\title{
Erratum
}

\section{Results from DroXo}

\section{The variability of fluorescent Fe $6.4 \mathrm{keV}$ emission in the young star Elias 29. High-energy electrons in the star's accretion tubes?}

\author{
G. Giardino ${ }^{1}$, F. Favata ${ }^{2}$, I. Pillitteri ${ }^{3}$, E. Flaccomio ${ }^{3}$, G. Micela ${ }^{3}$, and S. Sciortino ${ }^{3}$ \\ 1 ESA - Research and Science Support Department, ESTEC, Postbus 299, 2200 AG Noordwijk, The Netherlands \\ e-mail: ggiardin@rssd.esa.int \\ 2 ESA - Planning and Community Coordination Office, Science Programme, 8-10 rue Mario Nikis, 75738 Paris Cedex 15, France \\ 3 INAF - Osservatorio Astronomico di Palermo, Piazza del Parlamento 1, 90134 Palermo, Italy \\ A\&A, 475, 891-900 (2007), DOI: 10.1051/0004-6361:20077899
}

Key words. ISM: clouds - ISM: individual objects: $\rho$-Oph cloud - stars: pre-main sequence $-\mathrm{X}$-rays: stars stars: individual: Elias 29 - Errata, addenda

\section{Erratum}

In the original paper, the equivalent width of the Fe $6.4 \mathrm{keV}$ emission line from Elias 29 was incorrectly calculated, resulting in a systematic underestimate. The error was due to the incorrect use of the task EQWIDTH in XSPEC, the package used for the spectral analysis. By default, the command EQWIDTH computes the equivalent width of a Gaussian component using the level of the continuum within the energy range $0.95 E_{0}-1.05 E_{0}$, where $E_{0}$ is the position of the Gaussian line. In our case, $E_{0}=6.4 \mathrm{keV}$ and the continuum level was computed within $6.08-6.72 \mathrm{keV}$, which includes the strong Fe XXV emission line at $6.7 \mathrm{keV}$. Thus, the use of the XSPEC task in its default mode led us to systematically overestimate the level of the continuum and, by consequence, to underestimate the equivalent width of the Fe $6.4 \mathrm{keV}$ emission line. We provide here the correct equivalent width for the $6.4 \mathrm{keV}$ excess in the spectra of Elias 29 for each time segment. This was also derived with the EQWIDTH command in XSPEC but by limiting the energy range where the continuum level is derived to $0.99 E_{0}-1.01 E_{0}$, that is, $6.34-6.46 \mathrm{keV}$, thus excluding the strong Fe XXV emission line.

In addition, while repeating the spectral fits, a mistake in the calculation of the emission measure $(E M)$ was discovered. The new version of Tables 1-4 below give the correct equivalent width for the $6.4 \mathrm{keV}$ excess and the correct $E M$ values.

In the case of the fits to the PN data in the original paper, we provided the probability that the line could have been spurious, as derived by Montecarlo simulations (Table 3). The fits to the simulated data were repeated and the equivalent width of the $6.4 \mathrm{keV}$ emission computed again using the EQWIDTH command over the correct range. The new values of the derived probabilities in Table 3 are not significantly different from the old ones. The new value for the $6.4 \mathrm{keV}$ emission line equivalent width of the spectrum integrated over the time intervals "seg2", "seg3", "seg4", and "seg5" (Sect. 3 of the paper) is $W_{6.4 \mathrm{keV}}=354 \mathrm{eV}$.

\section{Conclusions}

As regrettable as these mistakes are, they do not change the conclusions of the paper. The error in the calculation of the $E M$ has no impact on any of the conclusions, since this quantity was only presented in the tables and was not used elsewhere in the paper. The equivalent width of the $\mathrm{Fe} 6.4 \mathrm{keV}$ emission line was discussed at several points in the paper; however, the mistake made in its calculation also does not change the conclusions of the paper. Indeed, as briefly outlined below, the new higher values reinforce some of the conclusions.

One of the main results of the paper is the observation of the time variability of the $\mathrm{Fe} 6.4 \mathrm{keV}$ emission, and this is not affected by the changes in the values of the line equivalent width; indeed, the newly calculated probabilities in Table 3 are still fully consistent with the $6.4 \mathrm{keV}$ emission being absent during the first segment of the observation. In the original paper we stressed that no fluorescence, whether from the photosphere or from a circumstellar shell, can result in the large equivalent widths observed, given a plausible thermal photoionising continuum. This is even truer given the new higher values. The higher values are also even more difficult to reconcile with emission from photoionisation of the material in the circumstellar disc. The model of George \& Fabian (1991) for a centrally illuminated face-on disc predicts a maximum equivalent width of only $120 \mathrm{eV}$. In their recent study of fluorescent Fe $\mathrm{K} \alpha$ emission, Drake et al. (2008) predicts equivalent width of the order of $150 \mathrm{eV}$ with the most favourable geometry for the illumination of an infinite flat disc, for a continuum temperature of $8 \mathrm{keV}$ (which is twice the temperature derived for Elias 29 continuum emission).

Our discussion of an alternative scenario where fluorescent emission is due to collisional excitation by a population of nonthermal electrons is also not affected by the change in the equivalent width of the $\mathrm{K} \alpha$ emission since this discussion was based on the observed line flux $\left(\sim 10^{-6}\right.$ photons $\left.\mathrm{cm}^{-2} \mathrm{~s}^{-1}\right)$, the value of which is unchanged.

\section{References}

Drake, J. J., Ercolano, B., \& Swartz, D. A. 2008, ApJ, 678, 385 George, I. M., \& Fabian, A. C. 1991, MNRAS, 249, 352 
Table 1. Best-fit parameters from the fits to the spectra of Elias 29, during 6 different time intervals (PN data only), using an absorbed 1T thermal model.

\begin{tabular}{lccccccc}
\hline \hline Time interval & $N(\mathrm{H})$ & $k T$ & $Z$ & $E M$ & $\chi^{2} /$ d.o.f. & $P$ & $F_{\mathrm{X}}$ \\
\hline & $N_{22}$ & $\mathrm{keV}$ & $Z_{\odot}$ & $E M_{53}$ & & & $F_{-13}$ \\
\hline seg1 & $5.7 \pm 0.7$ & $4.6 \pm 0.9$ & $1.0 \pm 0.3$ & $0.7 \pm 0.3$ & 1.0 & 0.6 & 1.8 \\
flare & $9.5 \pm 2.0$ & $4.1 \pm 1.5$ & $0.9 \pm 0.3$ & $4.5 \pm 3.4$ & 0.9 & 0.5 & 7.5 \\
seg2 & $8.1 \pm 1.3$ & $3.1 \pm 0.8$ & $1.0 \pm 0.3$ & $0.7 \pm 0.5$ & 1.5 & 0.0 & 1.1 \\
seg3 & $6.6 \pm 0.8$ & $3.4 \pm 0.7$ & $0.7 \pm 0.2$ & $0.8 \pm 0.4$ & 1.0 & 0.5 & 1.3 \\
seg4 & $7.7 \pm 0.6$ & $3.7 \pm 0.5$ & $0.7 \pm 0.1$ & $1.7 \pm 0.5$ & 1.4 & 0.0 & 2.7 \\
seg5 & $4.9 \pm 0.4$ & $4.7 \pm 0.8$ & $0.6 \pm 0.1$ & $1.1 \pm 0.3$ & 1.3 & 0.1 & 2.5 \\
\hline
\end{tabular}

Note: units are $N_{22}=10^{22} \mathrm{~cm}^{-2}, E M_{53}=10^{53} \mathrm{~cm}^{-3}$, and $F_{-13}=10^{-13} \mathrm{erg} \mathrm{cm}^{-2} \mathrm{~s}^{-1}$. The spectral fits were carried out in the energy range $1.0-9.0 \mathrm{keV}$. Flux values refer to energy range $1.0-7.5 \mathrm{keV}$. The error bars are at $1 \sigma$. Reduced $\chi^{2}$ and null-hypothesis probability $(P)$ for the fits are also given.

Table 2. Best-fit parameters from the joint fits to PN, MOS1 and, MOS2 spectra of Elias 29, during 5 different time intervals, using an absorbed 1T thermal model.

\begin{tabular}{lccccccc}
\hline \hline Time interval & $N(\mathrm{H})$ & $k T$ & $Z$ & $E M$ & $\chi^{2} /$ d.o.f. & $P$ & $F_{\mathrm{X}}$ \\
\hline & $N_{22}$ & $\mathrm{keV}$ & $Z_{\odot}$ & $E M_{53}$ & & & $F_{-13}$ \\
\hline seg1† & $5.5 \pm 0.5$ & $4.4 \pm 0.7$ & $1.0 \pm 0.2$ & $0.9 \pm 0.3$ & 1.1 & 0.1 & 2.1 \\
seg2 $\dagger$ & $7.4 \pm 0.8$ & $3.8 \pm 0.7$ & $0.8 \pm 0.2$ & $0.8 \pm 0.3$ & 1.6 & 0.0 & 1.3 \\
seg3 & $6.6 \pm 0.5$ & $3.5 \pm 0.5$ & $0.7 \pm 0.1$ & $1.1 \pm 0.3$ & 1.1 & 0.2 & 1.7 \\
seg4 & $7.5 \pm 0.4$ & $3.7 \pm 0.4$ & $0.7 \pm 0.1$ & $1.9 \pm 0.4$ & 1.5 & 0.0 & 3.0 \\
seg5 & $5.7 \pm 0.4$ & $4.0 \pm 0.5$ & $0.5 \pm 0.1$ & $1.5 \pm 0.4$ & 1.6 & 0.0 & 2.3 \\
\hline
\end{tabular}

$\dagger$ MOS1 data (mostly) unavailable for this time interval so only PN and MOS2 data were used.

Table 3. Best-fit parameters from the fits to the spectra of Elias 29, during 6 different time intervals (PN data only), using an absorbed 1T thermal model with an additional line at $6.4 \mathrm{keV}$ (Fe fluorescent line).

\begin{tabular}{|c|c|c|c|c|c|c|c|c|c|c|}
\hline Time interval & $N(\mathrm{H})$ & $k T$ & $Z$ & $E M$ & $\overline{f_{6.4 \mathrm{keV}}}$ & $W_{6.4 \mathrm{keV}}$ & $\chi^{2} /$ d.o.f. & $P$ & $F_{\mathrm{X}}$ & $R_{6.4 \mathrm{keV}} \ddagger$ \\
\hline & $N_{22}$ & $\mathrm{keV}$ & $Z_{\odot}$ & $E M_{53}$ & $f_{-6}$ & $\mathrm{eV}$ & & & $F_{-13}$ & $\%$ \\
\hline seg1 & $5.5 \pm 0.7$ & $4.8 \pm 1.0$ & $1.1 \pm 0.3$ & $0.7 \pm 0.3$ & $0.1 \pm 0.7$ & 34.8 & 1.0 & 0.5 & 1.8 & 46 \\
\hline flare & $9.4 \pm 2.0$ & $3.9 \pm 1.4$ & $0.9 \pm 0.3$ & $4.5 \pm 3.4$ & $2.0 \pm 5.9$ & 162.0 & 0.9 & 0.6 & 7.5 & 22 \\
\hline $\operatorname{seg} 2$ & $8.2 \pm 1.3$ & $2.8 \pm 0.6$ & $1.1 \pm 0.3$ & $0.8 \pm 0.4$ & $1.1 \pm 0.6$ & 834.0 & 1.3 & 0.1 & 1.1 & 0.1 \\
\hline seg3 & $6.6 \pm 0.8$ & $3.3 \pm 0.6$ & $0.7 \pm 0.2$ & $0.9 \pm 0.4$ & $0.5 \pm 0.5$ & 270.0 & 0.9 & 0.6 & 1.3 & 4.0 \\
\hline seg4 & $7.6 \pm 0.6$ & $3.6 \pm 0.4$ & $0.7 \pm 0.1$ & $1.7 \pm 0.5$ & $0.7 \pm 0.7$ & 171.0 & 1.3 & 0.1 & 2.7 & 3.1 \\
\hline $\operatorname{seg} 5$ & $4.9 \pm 0.4$ & $4.5 \pm 0.8$ & $0.6 \pm 0.1$ & $1.1 \pm 0.3$ & $1.1 \pm 0.8$ & 335.0 & 1.2 & 0.2 & 2.5 & 0.3 \\
\hline seg3+seg4+seg5 & $6.4 \pm 0.3$ & $3.9 \pm 0.3$ & $0.6 \pm 0.1$ & $1.2 \pm 0.2$ & $1.0 \pm 0.3$ & 330.0 & 1.3 & 0.0 & 2.1 & 0.0 \\
\hline
\end{tabular}

$\$$ Probability that a line with a best-fit value equal or greater than $W_{6.4} \mathrm{kev}$ could be due to a random fluctuation in the data, as estimated via Monte Carlo simulations (see Sects. 3 and 4).

$\diamond$ Results for the fit to the source's spectrum integrated over time intervals "seg3", "seg4", and "seg5".

Note: For the line, the total flux in the line $\left(f_{6.4 \mathrm{keV}}\right.$ in units of $f_{-6}=10^{-6}$ photons cm-2 $\left.\mathrm{s}^{-1}\right)$ and the equivalent width $\left(W_{6.4 \mathrm{keV}}\right)$ are given.

Table 4. Best-fit parameters from joint fits to PN, MOS1, and MOS2 spectra of Elias 29, during 5 different time intervals, using an absorbed 1T thermal model with an additional line at $6.4 \mathrm{keV}$ (Fe fluorescent line).

\begin{tabular}{lccccccccc}
\hline \hline Time interval & $N(\mathrm{H})$ & $k T$ & $Z$ & $E M$ & $f_{6.4 \mathrm{keV}}$ & $W_{6.4 \mathrm{keV}}$ & $\chi^{2} /$ d.o.f. & $P$ & $F_{\mathrm{X}}$ \\
\hline & $N_{22}$ & $\mathrm{keV}$ & $Z_{\odot}$ & $E M_{53}$ & $f_{-6}$ & $\mathrm{eV}$ & & & $F_{-13}$ \\
\hline seg1 $\dagger$ & $5.5 \pm 0.5$ & $4.3 \pm 0.8$ & $1.0 \pm 0.2$ & $0.9 \pm 0.3$ & $0.1 \pm 0.7$ & 26.6 & 1.2 & 0.1 & 2.1 \\
seg2 $\dagger$ & $7.5 \pm 0.8$ & $3.5 \pm 0.7$ & $0.9 \pm 0.2$ & $0.9 \pm 0.3$ & $1.1 \pm 0.7$ & 539.0 & 1.4 & 0.0 & 1.3 \\
seg3 & $6.7 \pm 0.6$ & $3.3 \pm 0.5$ & $0.7 \pm 0.2$ & $1.1 \pm 0.4$ & $0.4 \pm 0.6$ & 171.0 & 1.1 & 0.3 & 1.7 \\
seg4 & $7.5 \pm 0.4$ & $3.5 \pm 0.3$ & $0.7 \pm 0.1$ & $1.9 \pm 0.4$ & $1.2 \pm 0.7$ & 288.0 & 1.4 & 0.0 & 3.0 \\
seg5 & $5.8 \pm 0.4$ & $3.7 \pm 0.5$ & $0.5 \pm 0.1$ & $1.6 \pm 0.4$ & $1.2 \pm 0.7$ & 330.0 & 1.5 & 0.0 & 2.3 \\
\hline
\end{tabular}

$\dagger$ MOS1 data (mostly) unavailable for this time interval so only PN and MOS2 data were used. 